\title{
Temporal variation in the incidence of treated psychotic disorders in young people
}

\author{
Baptiste Pignon ${ }^{1}$, Scott Eaton ${ }^{2,3}$, Franck Schürhoff ${ }^{1}$, Andrei Szöke ${ }^{1}$, Patrick \\ McGorry ${ }^{2,3}$, Brian O’Donoghue ${ }^{2,3, *}$ \\ ${ }^{1}$ Univ Paris-Est-Créteil (UPEC), AP-HP, Hôpitaux Universitaires « H. \\ Mondor », DMU IMPACT, INSERM, IMRB, translational \\ Neuropsychiatry, Fondation FondaMental F-94010 Creteil, France Univ Paris-Est-Créteil (UPEC), AP- \\ HP, Hôpitaux Universitaires « $\mathrm{H}$. \\ Mondor », DMU IMPACT, INSERM, IMRB, translational \\ Neuropsychiatry, Fondation FondaMental F-94010 Creteil, France \\ ${ }^{2}$ Orygen, 35 Poplar rd, Parkville, Melbourne, Australia \\ ${ }^{3}$ Centre for Youth Mental Health, University of Melbourne, Melbourne, Australia
}

*Corresponding author:

Dr. Brian O’Donoghue

Consultant Psychiatrist

Orygen Youth Health, 35 Poplar rd, Parkville, VIC 3052

Phone: +61-3-93422800, Email: brian.odonoghue@ orygen.org.au 


\begin{abstract}

\section{Introduction}

The incidence of psychotic disorders varies between geographical areas, however less is known about whether it varies over time in the same region. Analyzing this temporal variation of incidence could improve the allocation of healthcare resources and our understanding of the aetiology of psychotic disorders. This study aimed to determine whether there was a change in the incidence of psychotic disorders over a six-year period.
\end{abstract}

\title{
Methods
}

Young people aged 15 to 24 presenting with a first episode of psychosis (FEP) attending an early intervention service in Melbourne between 2011 and 2016 were included. The population at-risk was determined from the two corresponding census periods and analyses were adjusted for age, sex and migrant status.

\section{Results}

A total of 1217 young people presented with a FEP over the six-year period and the crude incidence rate in 2011 was 102.4 per 100,000 population at-risk, compared to 125.4 in 2016 . There was an increase in the incidence by 33\% in 2015 (aIRR $=1.33,95 \%$ CI 1.09-1.63) and $28 \%$ in 2016 (aIRR $=1.28,95 \%$ CI 1.05-1.56). When examined according to diagnostic groups, there was an increase in the incidence of substance-induced psychotic disorders among females in 2015 (aIRR=4.62, 95\% CI 1.02-20.8).

\section{Discussion}

This study shows significant temporal variations in the incidence of treated psychotic disorders. These findings demonstrate that early intervention services should continually monitor incidence case numbers and funding should be provided accordingly, to ensure the required intensive and comprehensive treatments can be sustained. 


\section{Introduction}

Epidemiologic studies of psychotic disorders have shown that incidence vary spatially, e.g. according to the latitude, between regions within a country, or between neighborhoods within a city (McGrath et al., 2004; Saha et al., 2006; Szöke et al., 2016; O’Donoghue et al., 2016; Jongsma et al., 2018). These variations are associated with several factors, such as the level of urbanicity, social deprivation, or socio-economic level (Colodro-Conde et al., 2018; Kirkbride et al., 2014; March et al., 2008; Richardson et al., 2018).

Studies of temporal variations in the incidence of psychotic disorders are much less numerous. Castle et al. (1991) analyzed the evolution of the incidence of different psychotic disorders in Camberwell (London) between 1965 and 1984 and found an increase (19.9 per 100,000 persons per year in 1964-69, 24.9 in 1980-84), while in Nottingham, Kirkbride et al. (2009) found that there was no overall difference between 1978 and 1999, however there was an increase of substance-induced psychotic disorders. In Taiwan, first hospital admission for people with a diagnosis of schizophrenia decreased between 1998 and 2007 (Chiang et al., 2017), and this was replicated in Israel (Levinson and Lerner, 2013). However, it is likely that these findings reflected he transition to community-based treatment, as opposed to a decline in the incidence of psychotic disorders.

Analyzing the temporal variations in the incidence of psychotic disorder is of significant importance given the necessity to appropriately allocate healthcare resources over extended periods of time. The optimal allocation of healthcare resources is of particular importantance in the early care of psychotic disorders as providing appropriate and personalized care for people with a first episode of psychotic disorders (FEP) is critical for their prognosis (Fusar- Poli et al., 2017). Moreover, changes in the incidence of psychotic disorder in certain areas and the analyses of the determinants of this evolution could bring insights into the aetiology of psychotic disorders (Kirkbride et al., 2009).

The aim of the present study was to determine the incidence of treated psychotic disorders within a defined catchment area over a six-year period and to determine whether there was a change in incidence across this time period. It was also aimed to determine whether the incidence of FEP varied according to sex and different diagnostic groups. Post-hoc analysis was also performed to determine whether the clinical characteristics of the cohort varied over 
time as this may have contributed to the observed changes in the incidence of FEP. Four clinical characteristics were examined: the duration of untreated psychosis (DUP), cannabis and methamphetamine abuse and the proportion of first-generation migrants.

\section{Methods}

\section{Settings and participants}

The Early Psychosis Prevention and Intervention Centre (EPPIC) is an Early intervention (EI) in psychosis service within the Orygen Specialist Program, the state-based youth mental health service for people aged between 15 and 24. The EPPIC service was one of the first EI services in the world and was established in 1992 (McGorry et al., 1996). The catchment area covers 59 postcodes in the north-western and western areas of Melbourne, Australia, some of which have high levels of social deprivation and social fragmentation (Eaton et al., 2019).

All young people with a FEP aged 15 to 24 years-old, living in the catchment area and attending the EPPIC service between 01.01.2011 and 31.12.2016, were included in the study (Eaton et al., 2019; Pignon et al., 2019). In order to reduce help-seeking delays, there is an open referral system, in that young people can be referred by anyone, including themselves and the common sources of referral are family, general practitioners, school and university counsellors, private psychologists and youth workers. EPPIC is publicly funded and is the only such service that provides treatment for FEP in the catchment area. It is possible that individuals are referred directly to private providers; however, as the EI service is well established within the area and well known to professional referrers, it is unlikely that many individuals are referred to an alternative service. There were no changes to the criteria or procedures for entry to the service during the study period.

\section{Demographic and Clinical Information}

Diagnoses of psychotic disorders and concurrent diagnoses, such as substance abuse disorders, were made by the treating consultant psychiatrist at three months after service entry and reviewed at discharge according to DSM-IV classification of mental disorders. This method and criteria were consistent throughout the study period. A FEP was determined by the presence of full threshold psychotic symptoms that are present daily for at least one week and result in distress or an associated negative consequence on functioning. Diagnoses were grouped according to schizophrenia-spectrum disorders (schizophrenia, schizophreniform 
disorder and schizoaffective disorder), affective psychotic disorders (bipolar affective disorder and depression with psychosis), substance-induced psychotic disorders, and other psychotic disorders (brief psychotic disorder, delusional disorder, psychosis NOS). Details pertaining to country of birth were obtained from a standard registration form and therefore it could be determined if individuals were first-generation migrants. Information pertaining to parental place of birth was not available and therefore second-generation migrants could not be identified. Information on the prevalence of substance use was obtained from the clinical file and this was recorded systematically on entry to service. Furthermore, an estimate of the onset of psychotic symptoms was obtained and used to determine the DUP.

\section{Census data}

Census data was available for the defined catchment area of 59 postcodes for 2011 and 2016, corresponding to the starting and end point of the study period. In order to estimate the at-risk population in the catchment area for each year, the differences in population between the time periods (2011-2016) were calculated and divided by five (this represented the estimated yearly variation in population) and then this was added to the population of each previous year. This method of analysis assumed that there was linear population growth between 2011 and 2016, therefore we also estimated the population at risk based on exponential population growth and repeated the analysis. For each postcode, population data was available pertaining to age category (15-19, 20-24), sex and place of birth (thus first-generation migrant status) and therefore it was possible to control for these factors in the analysis.

\section{Statistical analysis}

The incidence rate ratios (IRRs) of treated psychotic disorders among people aged between 15 and 24 for each year from 2012 and 2016 were compared to the IRR from the reference year 2011 using Poisson regression in STATA I/C v15.1 (StataCorp., 2017). Comparisons were adjusted on age, sex and first-generation migrant status. Secondarily, the same comparisons were done for each of the four diagnosis categories (schizophrenia-spectrum disorders, affective psychotic disorders, and substance-induced psychotic disorder, other psychotic disorders). It was assessed whether effect modification was present between time and sex, i.e., whether an association between time and the incidence of FEP was different for men and women. The model adjusted for age, sex and migrant status was compared to the same model with an interaction term fitted between sex and year, and a likelihood ratio test was performed between the two models. A $\mathrm{p}<0.05$ was taken as evidence that effect modification was 
present. As the use of the first year (2011) as the reference value was arbitrary, we repeated the analyses using each different year as the reference value.

\section{Results}

\section{Participants}

A total of 1217 young people presented with a FEP during the six-year study period between 2011 and 2016. The mean age was 19.6 years $(\mathrm{sd}= \pm 2.8)$. A total of $58.5 \%(\mathrm{n}=712)$ were male and the majority were single (93.5\%). In regard to diagnosis, $42.0 \%$ had diagnoses of schizophrenia-spectrum disorders, while $21.4 \%$ had affective psychotic disorders, and $12.4 \%$ substance-induced psychotic disorders. More than half of the young people had concurrent cannabis abuse (52.2\%) and $27.7 \%$ had methamphetamine abuse. Details on the sociodemographic and clinical characteristics of the cohort are presented in Table $\mathbf{1}$ and information on these factors across the six years (Supplementary Table 1).

\section{Incidence of first-episode psychosis across six years}

In 2011, there were 160 cases of treated FEP from an at-risk population of 156,280 people aged 15 to 24 and this corresponds to a crude incidence rate of 102.4 per 100,000 population at-risk (see census data in Supplementary Table 2). In 2016, there were 240 cases of FEP from an at-risk population of 191,356 and this corresponds to a crude incidence rate of 125.4 per 100,000 population at risk. Using the incidence rate of 2011 as a reference range, there was a significant increase in the incidence rate of FEP in 2015 (aIRR $=1.33$, 95\% CI 1.09-1.63, $\mathrm{p}<0.01$ ) and 2016 (aIRR=1.28, 95\% CI 1.05-1.56, $\mathrm{p}=0.02$ ) when adjusted for age, sex and migrant status. The incidence rate ratios for the individual years from 2012 to 2016 are presented in Table 2 for the total cohort. This analysis was repeated with the at-risk population based on exponential growth, and the results showed similar patterns (Supplementary Table 3). Similarly, the analysis was repeated with the different years as reference year and these results are presented in Supplementary Table 4.

The incidence rates according to sex are presented in Table 2 and an increase of over $50 \%$ in the incidence rate of FEP among females was observed in 2013 (aIRR=1.59, 95\% CI 1.152.20, p<0.01), 2015 and 2016 ( $\mathrm{aIRR}=1.58,95 \%$ CI 1.15-2.17, p<0.01 for both). However, 
there was no evidence of a statistically significant interaction between year and sex on the incidence of FEP $(\mathrm{p}=0.25)$.

When examined according to the different diagnosis categories, there was no difference in the incidence rates in the six years in those with a diagnosis of a schizophrenia-spectrum disorders, affective psychotic disorders or substance-induced psychotic disorder (Table 3). There were trend level increases in the incidence rates for those with a diagnosis of substanceinduced psychotic disorders, specifically in 2015 (aIRR=1.74, 95\% CI 0.97-3.10, p=0.06) and $2016(\mathrm{aIRR}=1.72,95 \%$ CI 0.97-3.06, $\mathrm{p}=0.06)$.

\section{Post-hoc analysis}

As a result of these observations, post-hoc analysis was performed to examine the yearly incidence of substance-induced psychotic disorders according to sex. This analysis found that in 2015 there was an increase in the incidence of substance-induced psychotic disorders among females compared to 2011 (aIRR=4.62, 95\% CI 1.02-20.84, p=0.05, see Supplementary Table 5 for details).

Further post-hoc analysis was also conducted in order to identify clinical factors that could having contributed to the observed change in the incidence of psychotic disorders over the six years. The prevalence of four factors, specifically the DUP, cannabis and methamphetamine abuse and proportion of first-generation migrants was examined across the six years of the study. A change in the DUP was observed across the six years, with a median DUP of 4 weeks (intervalley quartile range (IQR): 2-14 weeks) in 2011 increasing to 12 weeks in 2015 (IQR: 3-52 weeks) and 2016 (IQR: 2-52 weeks) (Kruskal-Wallis $\mathrm{H}=21.3$, df=5, p<0.01). This finding was present for both males and females. There was a difference in the proportion of female migrants over the six-year period $\left(\chi^{2}=15.5, \mathrm{df}=5, \mathrm{p}<0.01\right)$ which was most likely due to a low proportion in $2014(6.8 \%)$, compared with the other years' ranges of 20-30\%. There was no difference in the proportion of people using cannabis or methamphetamine (see details in Supplementary Table 1).

\section{Discussion}

Summary of findings 
This study has demonstrated that the incidence of treated psychotic disorders is not temporally stable, as in comparison to 2011, there was an increase in the incidence in 2015 and 2016. In these years, the incidence rate in the catchment area increased by 33\% in 2015 and $28 \%$ in 2016.

\section{Comparison to previous literature}

Comparisons with other studies are difficult as the incidence may vary according to local factors, such as the rate of cannabis use, or proportions of migrants, or according to mental health institutions organization (Di Forti et al., 2019; Kirkbride et al., 2012; Selten et al., 2020). Overall, other studies of the evolution of the incidence of psychotic disorders during the last decades found a decrease in incidence. For instance, a study of the evolution of firstadmission rates for schizophrenia in Taiwan between 1998 and 2007 showed a biphasic change, with an increase until 2001, which was then followed with a decrease (Chiang et al., 2017). Although, this study examined admissions rates, as opposed to the incidence and this could have changed as a result of de-institutionalization. Consistent with the findings from Taiwan, Nielsen et al. (2010) found that the incidence cases of psychotic disorders decreased in Denmark between 1996 and 2005 by 17\%. In Nottingham, Kirkbride et al. (2009) found that the incidence of psychotic disorders was stable over two decades, although there was variations in the diagnoses. Interestingly, the study found an increase in the incidence of substance-induced psychotic disorders, which reflects the findings in our study. The authors hypothesized that this increase in substance-induced psychotic disorders may have been related to an increase in the potency of the cannabis, that had been observed in the catchment area (Bone and Waldron, 1997).

\section{Possible explanations for the findings}

An explanation for the increase in the incidence of psychotic disorders within the catchment area may be related to an increase in the prevalence of established risk factors. For example, the potency of cannabis has increased over the last number of years in Australia (Hall and Swift, 2000; Swift et al., 2013) and this is correlated with the risk for developing a psychotic disorder. A recent European study on the contribution of cannabis use to the variations of the incidence of psychotic disorders across five European countries and Brazil showed that the population attributable fraction of daily use of high-potency cannabis reached $35.9 \%$ in Paris (France), 51.5\% in London (UK), or 69.6\% in Amsterdam (Netherlands) (Di Forti et al., 2019). This hypothesis of an increase of the potency of the cannabis used in the catchment 
area could explain the fact that the substance-induced psychotic disorders increase in 2015 and 2016 (Table 3 and Supplementary Table 5: increases of around 70\%, although these increases did not reach significance in the total cohort, contrary to the female sub-group) without significant changes in cannabis or methamphetamine abuses (Supplementary Table 1). It is curious that this increase was observed in females and it could reflect a greater vulnerability to developing substance induced psychotic disorders. Interestingly, the National Drug Strategy Household survey, which is conducted in Australia every three years, found that there had been an increase in illicit drug use in females from 2013 to 2016 (Australian Institute of Health and Welfare (AIHW), 2017).

There is another possible mechanism by which cannabis could increase the incidence of treated psychotic disorders in those aged 15 to 24 . The use of cannabis is associated with an earlier age of onset of a FEP, a meta-analysis indicated this is by nearly three years (Large et al., 2011) and a more recent individual study demonstrated a difference by nearly six years (O’Donoghue et al., 2015). There are two hypotheses underlying these findings. The first is that cannabis precipitates the psychotic disorder and results in an earlier expression of a disorder that would have occurred anyway at a later stage (Haney and Evins, 2016). The second is that the use of cannabis results in new incidence cases of FEP that would have not developed in the absence of cannabis. In the first scenario, the incidence of FEP would be increased in the age group included in this study (15 to 24); however, it would be decreased in the older age groups. In the second scenario, the incidence of FEP would be increased in the age groups in which cannabis use is prevalent.

A similar phenomenon could be observed from the introduction of EI for psychosis services. As these services are established and the strategies to reduce the delays to treatment are employed, the delay for individuals receiving the appropriate treatment can be substantially reduced (Krstev et al., 2004). Furthermore, in the early stages of implementation, EI services identify a significant number of individuals with a very long DUP and this proportion decreases over time (O’Donoghue et al., 2014). Therefore, over time, EI services could see a reduction in the age of those who present with a FEP, as the gap between the age at onset and presentation decreases. However, this was not observed in this study, as the DUP was very low at the beginning of the study (4 weeks), most likely as a result of being a well-established EI service and while the DUP fluctuated throughout the study period, it reached a maximum of 12 weeks and this would not explain more people presenting at a younger age. 
Another factor that is associated with the incidence of psychotic disorders is population density and urbanicity (Vassos et al., 2012) and as demonstrated by the census data, in the defined catchment area, the population of young people aged 15 to 24 increased by over 35,000 in five years and this represents an increase of over $22 \%$ during this time. As the catchment area did not change, this would have resulted in a significant increase in population density.

\section{Clinical implications}

Overall, this study demonstrates that the incidence of treated psychotic disorders is not temporally stable and changes over time. Mental health services have traditionally been funded on a per-capita basis however it has been argued that this distributes resources on an inequitable basis, as there are higher rates of psychotic disorders in more deprived areas and that have a higher proportion of migrants (O’Donoghue et al., 2016; Selten et al., 2020). As we know that these factors are dynamic over time, it makes sense that the incidence of psychotic disorders also changes over time. For example, the increase in the incidence of psychotic disorders observed in Camberwell between 1965 and 1984 was attributed to an influx of individuals of Afro-Caribbean ethnicity (Castle et al., 1991). Therefore, in addition to taking environmental factors into account to predict the expected incidence of psychotic disorders, service planners also need to consider the potential change of these factors over time.

This change in incidence rate of treated psychotic disorders could also lead to insights into aetiological factors in psychotic disorders. To date, the established risk factors for psychotic disorders, specifically cannabis use and migration have been examined, but there are other potential, unknown risk factors that could be identified if a change in these factors correlates with the change in incidence. For example, the availability of green spaces in residential areas is being examined as to whether it is associated with the incidence of psychotic disorders (Engemann et al., 2020). Moreover, the fact that the incidence of psychotic disorders increase solely among women should be examined further. For instance, the role of specific risk factors among women (or the decrease of the effect of protective factors) could explain this result (Riecher-Rössler, 2017).

\section{Strengths and Limitations}


The findings of this study need to be considered within the limitations of the design. However, as with most incidence of psychotic disorder studies (McGrath et al., 2004), only the treated cases were considered. Thus, individuals without treatment, or with onset of psychotic disorders between 15 and 24 years-old but who received treatment after 24 yearsold, were not included. Missed incident cases phenomenon has been showed to bias incidence studies (Pignon et al., 2018). Therefore, it is possible that the increase in the incidence could also be due to improved detection of FEP. A further limitation is that we only controlled for age across two age categories (15-19 and 20-24) and we also used a dichotomous variable for first-generation migrant status, despite it being established that different migrant groups in Australia have different levels of risk for psychotic disorders (O'Donoghue et al., 2020). Furthermore, as the main finding of this study is that incidence increased among females, it is a limitation to the study that we only had information pertaining to sex and that we did not have information on the gender by which the young person may have identified with.

\section{Conclusions}

The incidence of treated psychotic disorder varies according to geographical areas and this study provides evidence that the incidence also varies temporally. Therefore, traditional methods of funding of mental health services on a per capita basis need to be replaced by a more sophisticated system that incorporates the characteristics of the catchment areas and the temporal variation in the incidence of psychotic disorders, which from this study, appear to increase over time. 


\section{References}

Australian Institute of Health and Welfare (AIHW), 2017. National Drug Strategy Household Survey (NDSHS) 2016-key findings, Illicit use of drugs [WWW Document]. Aust. Inst. Health Welf. URL https://www.aihw.gov.au/reports/illicit-use-of-drugs/ndshs-2016-keyfindings/contents/illicit-use-of-drugs (accessed 3.5.21).

Bone, C., Waldron, S., 1997. New trends in illicit cannabis cultivation in the United Kingdom of Great Britain and Northern Ireland (1 - 1006 No. Bulletin on Narcotics). UNODC.

Castle, D., Wessely, S., Der, G., Murray, R.M., 1991. The incidence of operationally defined schizophrenia in Camberwell, 1965-84. Br. J. Psychiatry J. Ment. Sci. 159, 790-794.

Chiang, C.-L., Chen, P.-C., Huang, L.-Y., Kuo, P.-H., Tung, Y.-C., Liu, C.-C., Chen, W.J., 2017. Time trends in first admission rates for schizophrenia and other psychotic disorders in Taiwan, 1998-2007: a 10-year population-based cohort study. Soc. Psychiatry Psychiatr. Epidemiol. 52, 163-173.

Colodro-Conde, L., Couvy-Duchesne, B., Whitfield, J.B., Streit, F., Gordon, S., Kemper, K.E., Yengo, L., Zheng, Z., Trzaskowski, M., de Zeeuw, E.L., Nivard, M.G., Das, M., Neale, R.E., MacGregor, S., Olsen, C.M., Whiteman, D.C., Boomsma, D.I., Yang, J., Rietschel, M., McGrath, J.J., Medland, S.E., Martin, N.G., 2018. Association Between Population Density and Genetic Risk for Schizophrenia. JAMA Psychiatry 75, 901-910.

Di Forti, M., Quattrone, D., Freeman, T.P., Tripoli, G., Gayer-Anderson, C., Quigley, H., Rodriguez, V., Jongsma, H.E., Ferraro, L., La Cascia, C., La Barbera, D., Tarricone, I., Berardi, D., Szöke, A., Arango, C., Tortelli, A., Velthorst, E., Bernardo, Miguel, Del-Ben, C.M., Menezes, P.R., Selten, J.-P., Jones, P.B., Kirkbride, J.B., Rutten, B.P., de Haan, L., Sham, P.C., van Os, J., Lewis, C.M., Lynskey, M., Morgan, C., Murray, R.M., Amoretti, S., Arrojo, M., Baudin, G., Beards, S., Bernardo, Miquel, Bobes, J., Bonetto, C., Cabrera, B., Carracedo, A., Charpeaud, T., Costas, J., Cristofalo, D., Cuadrado, P., Díaz-Caneja, C.M., Ferchiou, A., Franke, N., Frijda, F., García Bernardo, E., Garcia-Portilla, P., González, E., Hubbard, K., Jamain, S., Jiménez-López, E., Leboyer, M., López Montoya, G., LorenteRovira, E., Marcelino Loureiro, C., Marrazzo, G., Martínez, C., Matteis, M., Messchaart, E., Moltó, M.D., Nacher, J., Olmeda, M.S., Parellada, M., González Peñas, J., Pignon, B., Rapado, M., Richard, J.-R., Rodríguez Solano, J.J., Roldán Díaz, L., Ruggeri, M., Sáiz, P.A., Sánchez, E., Sanjuán, J., Sartorio, C., Schürhoff, F., Seminerio, F., Shuhama, R., Sideli, L., 
Stilo, S.A., Termorshuizen, F., Tosato, S., Tronche, A.-M., van Dam, D., van der Ven, E., 2019. The contribution of cannabis use to variation in the incidence of psychotic disorder across Europe (EU-GEI): a multicentre case-control study. Lancet Psychiatry 6, 427-436.

Eaton, S., Harrap, B., Downey, L., Thien, K., Bowtell, M., Bardell-Williams, M., Ratheesh, A., McGorry, P., O’Donoghue, B., 2019. Incidence of treated first episode psychosis from an Australian early intervention service and its association with neighbourhood characteristics. Schizophr. Res. 209, 206-211.

Engemann, K., Pedersen, C.B., Agerbo, E., Arge, L., Børglum, A.D., Erikstrup, C., Hertel, O., Hougaard, D.M., McGrath, J.J., Mors, O., Mortensen, P.B., Nordentoft, M., Sabel, C.E., Sigsgaard, T., Tsirogiannis, C., Vilhjálmsson, B.J., Werge, T., Svenning, J.-C., Horsdal, H.T., 2020. Association Between Childhood Green Space, Genetic Liability, and the Incidence of Schizophrenia. Schizophr. Bull. sbaa058. https://doi.org/10.1093/schbul/sbaa058

Fusar- Poli, P., McGorry, P.D., Kane, J.M., 2017. Improving outcomes of first-episode psychosis: an overview. World Psychiatry 16, 251-265. https://doi.org/10.1002/wps.20446

Hall, W., Swift, W., 2000. The THC content of cannabis in Australia: evidence and implications. Aust. N. Z. J. Public Health 24, 503-508.

Haney, M., Evins, A.E., 2016. Does Cannabis Cause, Exacerbate or Ameliorate Psychiatric Disorders? An Oversimplified Debate Discussed. Neuropsychopharmacology 41, 393-401.

Jongsma, H.E., Gayer-Anderson, C., Lasalvia, A., Quattrone, D., Mulè, A., Szöke, A., Selten, J.-P., Turner, C., Arango, C., Tarricone, I., Berardi, D., Tortelli, A., Llorca, P.-M., Haan, L. de, Bobes, J., Bernardo, M., Sanjuán, J., Santos, J.L., Arrojo, M., Del-Ben, C.M., Menezes, P.R., Murray, R.M., Rutten, B.P., Jones, P.B., van Os, J., Morgan, C., Kirkbride, J.B., 2018. Treated incidence of psychotic disorders in the multinational EU-GEI study. JAMA Psychiatry 75, 36-46.

Kirkbride, J.B., Croudace, T., Brewin, J., Donoghue, K., Mason, P., Glazebrook, C., Medley, I., Harrison, G., Cooper, J.E., Doody, G.A., Jones, P.B., 2009. Is the incidence of psychotic disorder in decline? Epidemiological evidence from two decades of research. Int. J. Epidemiol. 38, 1255-1264.

Kirkbride, J.B., Jones, P.B., Ullrich, S., Coid, J.W., 2014. Social deprivation, inequality, and 
the neighborhood-level incidence of psychotic syndromes in East London. Schizophr. Bull. 40, 169-180.

Kirkbride, J.B., Stubbins, C., Jones, P.B., 2012. Psychosis incidence through the prism of early intervention services. Br. J. Psychiatry J. Ment. Sci. 200, 156-157.

Krstev, H., Carbone, S., Harrigan, S.M., Curry, C., Elkins, K., McGorry, P.D., 2004. Early intervention in first-episode psychosis--the impact of a community development campaign. Soc. Psychiatry Psychiatr. Epidemiol. 39, 711-719.

Large, M., Sharma, S., Compton, M.T., Slade, T., Nielssen, O., 2011. Cannabis use and earlier onset of psychosis: a systematic meta-analysis. Arch. Gen. Psychiatry 68, 555-561.

Levinson, D., Lerner, Y., 2013. Hospitalization of patients with schizophrenic and affective disorders in Israel in the aftermath of the structural and rehabilitation reforms. Isr. J. Health Policy Res. 2, 29.

March, D., Hatch, S.L., Morgan, C., Kirkbride, J.B., Bresnahan, M., Fearon, P., Susser, E., 2008. Psychosis and place. Epidemiol. Rev. 30, 84-100.

McGorry, P.D., Edwards, J., Mihalopoulos, C., Harrigan, S.M., Jackson, H.J., 1996. EPPIC: An Evolving System of Early Detection and Optimal Management. Schizophr. Bull. 22, 305326.

McGrath, J.J., Saha, S., Welham, J., El Saadi, O., MacCauley, C., Chant, D.C., 2004. A systematic review of the incidence of schizophrenia: the distribution of rates and the influence of sex, urbanicity, migrant status and methodology. BMC Med. 2, 13.

Nielsen, J., Quach, P.L., Emborg, C., Foldager, L., Correll, C.U., 2010. 10-Year trends in the treatment and outcomes of patients with first-episode schizophrenia. Acta Psychiatr. Scand. $122,356-366$.

O’Donoghue, B., Downey, L., Eaton, S., Mifsud, N., Kirkbride, J.B., McGorry, P., 2020. Risk of psychotic disorders in migrants to Australia. Psychol. Med. in press, 1-9. https://doi.org/10.1017/S0033291719004100

O’Donoghue, B., Lyne, J., Kinsella, A., Turner, N., O’Callaghan, E., Clarke, M., 2014. Detection and characteristics of individuals with a very long duration of untreated psychosis 
in an early intervention for psychosis service. Early Interv. Psychiatry 8, 332-339.

O’Donoghue, B., Lyne, J., Madigan, K., Lane, A., Turner, N., O’Callaghan, E., Clarke, M., 2015. Environmental factors and the age at onset in first episode psychosis. Schizophr. Res. 168, 106-112.

O’Donoghue, B., Lyne, J.P., Renwick, L., Lane, A., Madigan, K., Staines, A., O'Callaghan, E., Clarke, M., 2016. Neighbourhood characteristics and the incidence of first-episode psychosis and duration of untreated psychosis. Psychol. Med. 46, 1367-1378.

O’Donoghue, Brian, Roche, E., Lane, A., 2016. Neighbourhood level social deprivation and the risk of psychotic disorders: a systematic review. Soc. Psychiatry Psychiatr. Epidemiol. 51, 941-950.

Pignon, B., Eaton, S., Schürhoff, F., Szöke, A., McGorry, P., O’Donoghue, B., 2019. Residential social drift in the two years following a first episode of psychosis. Schizophr. Res. 210, 323-5.

Pignon, B., Schürhoff, F., Baudin, G., Tortelli, A., Ferchiou, A., Saba, G., Richard, J.-R., Pelissolo, A., Leboyer, M., Szöke, A., 2018. Relationship between incidence and prevalence in psychotic disorders: An incidence-prevalence-mortality model. Int. J. Methods Psychiatr. Res. e1719. https://doi.org/10.1002/mpr.1719

Richardson, L., Hameed, Y., Perez, J., Jones, P.B., Kirkbride, J.B., 2018. Association of Environment With the Risk of Developing Psychotic Disorders in Rural Populations: Findings from the Social Epidemiology of Psychoses in East Anglia Study. JAMA Psychiatry 75, 7583.

Riecher-Rössler, A., 2017. Oestrogens, prolactin, hypothalamic-pituitary-gonadal axis, and schizophrenic psychoses. Lancet Psychiatry 4, 63-72.

Saha, S., Chant, D.C., Welham, J.L., McGrath, J.J., 2006. The incidence and prevalence of schizophrenia varies with latitude. Acta Psychiatr. Scand. 114, 36-39.

Selten, J.-P., van der Ven, E., Termorshuizen, F., 2020. Migration and psychosis: a metaanalysis of incidence studies. Psychol. Med. 50, 303-13.

StataCorp., 2017. Stata Statistical Software: Release 15. Coll. Stn. TX StataCorp LLC. 
Swift, W., Wong, A., Li, K.M., Arnold, J.C., McGregor, I.S., 2013. Analysis of Cannabis Seizures in NSW, Australia: Cannabis Potency and Cannabinoid Profile. PLoS ONE 8.

Szöke, A., Pignon, B., Baudin, G., Tortelli, A., Richard, J.-R., Leboyer, M., Schürhoff, F., 2016. Small area-level variation in the incidence of psychotic disorders in an urban area in France: an ecological study. Soc. Psychiatry Psychiatr. Epidemiol. 51, 951-960.

Vassos, E., Pedersen, C.B., Murray, R.M., Collier, D.A., Lewis, C.M., 2012. Meta-analysis of the association of urbanicity with schizophrenia. Schizophr. Bull. 38, 1118-1123. 